\title{
MDCT-QCT, QUS, and DXA in healthy adults: An intermodality comparison
}

\author{
Malakeh Malekzadeh ${ }^{1}$, Mojgan Asadi ${ }^{2,3}$, Shahrokh Abbasi-Rad ${ }^{4,5}$, Jamileh Abolghasemi ${ }^{6}$, \\ Zohreh Hamidi ${ }^{3}$, Mahsa Talebi ${ }^{4,5}$, Mohammad Bagher Shiran*7,1, Hamidreza Saligheh Rad ${ }^{4,5}$
}

\section{Abstract}

Background: Cortical deceleration is the main reason for bone loss at peripheral sites. It was suggested that when peripheral bones were assessed for osteoporosis, management and therapy can be administered early. The main aim of this study was to assess the relationships between the central and peripheral measurements at different skeleton bone sites (spine, femur, forearm, tibia, and calcaneus) with available modalities: DXA, QUS, and MDCT-QCT.

Methods: The volunteers recruited in this study did not have any history or evidence of metabolic bone disease. Blood test and DXA measurements were used as inclusion criteria to select 40 healthy participants. The selected volunteers underwent 3 imaging modalities: QCT, DXA, and QUS. DXA-based measurements were made on 3 sites, including spine, femur, and forearm. QCT and QUS measurements were done for distal of tibia and calcaneus bones, respectively. The extracted parameters from the 3 modalities were analyzed using a bivariate (Pearson) correlation (r) in statistical software.

Results: The results showed moderate to good correlations between spongy bones in central and peripheral sites from all the modalities. However, there was no correlation between MDCT measures and central bone values. According to correlations between different peripheral sits, aBMD of $33 \%$ radius and trabecular vBMD in $38 \%$ distal tibia showed weak but significant relationship between peripheral bones $(\mathrm{r}=-0.342, \mathrm{p}=0.044)$.

Conclusion: The findings demonstrated how bones in central and peripheral sites were correlated. Multimodality imaging was used in this group of healthy volunteers. Also, it was found that QCT-based MDCT needs more optimization and requires further investigations.

Keywords: Multidetector computed tomography, Quantitative computed tomography, Quantitative ultrasound, bone, Normal adult

Conflicts of Interest: None declared

Funding: Iran University of Medical Sciences (Grant \# 96-0130-29826)

\section{*This work has been published under CC BY-NC-SA 1.0 license.}

Copyright $\odot$ Iran University of Medical Sciences

Cite this article as: Malekzadeh M, Asadi M, Abbasi-Rad Sh, Abolghasemi J, Hamidi Z, Talebi M, Shiran MB, Saligheh Rad H. MDCT-QCT, QUS, and DXA in healthy adults: An intermodality comparison. Med J Islam Repub Iran. 2019 (28 Dec);33:156. https://doi.org/10.47176/mjiri.33.156

\section{Introduction}

Bone has a complex structure that can be studied both at the macrostructure (cortical and trabecular bone) and microstructural level (Haversian system). One of the main bone diseases due to aging is osteoporosis, which is defined by the World Health Organization (WHO) (1994) as follows: a disease characterized by low bone mass and microarchitectural deterioration of bone tissue leading to

Corresponding author: Dr Mohammad Bagher Shiran, shiran.m@iums.ac.ir

1. Medical Physics Department, School of Medicine, Iran University of Medical Sciences, Tehran, Iran

2. Osteoporosis Research Center, Endocrinology and Metabolism Clinical Sciences Institute, Tehran University of Medical Sciences, Tehran, Iran

3. Endocrinology and Metabolism Research Center, Endocrinology and Metabolism Clinical Sciences Institute, Tehran University of Medical Sciences, Tehran, Iran

4. Quantitative Medical Imaging Systems Group, Research Center for Molecular and Cellula Imaging, Tehran University of Medical Sciences, Tehran, Iran

5. Medical Physics and Biomedical Engineering Department, Tehran University of Medical Sciences, Tehran, Iran

6. Department of Biostatistics, School of public health, Iran University of Medical Sciences, Tehran, Iran

7. Finetech in Medicine Research Center, Iran University of Medical Sciences, Tehran, Iran enhanced bone fragility and a consequent increase in fracture risk (1). Bone mineral density (BMD) quantification is often used for the diagnosis of osteoporosis. It is evident that older patients may have a 10 -fold-increased fracture risk with the same BMD compared to younger individuals $(2,3)$. It can be seen in clinics that BMD ( as a gold standard) is not a good marker in many cases, since

$\uparrow$ What is “already known” in this topic:

Bone loss occurs both in central spongy bones and in the peripheral cortical bones.

$\rightarrow$ What this article adds:

In this study, the correlation between volume BMD $\left(\mathrm{K}_{2} \mathrm{HPO}_{4} \mathrm{mg} / \mathrm{cc}\right)$ with other common modality values was examined based on multidetector computed tomography. 
there are some exceptions for some patients who have normal BMD but may have a fracture in the near future $(4,5)$.

Since the main reason for bone loss at peripheral sites is related to cortical deceleration due to remodeling within the cortex bone close to the bone marrow (6), adding other modalities to measure (quantity and quality approach) the peripheral sites may improve diagnosis. It is also suggested that when peripheral bones (distal radius and calcaneus) are assessed for osteoporosis, management and therapy can be administered earlier (7).

In this study, the main aim was to show the relationship between the central and peripheral bone measurements in different skeleton bones (including spine, femur, forearm, tibia, and calcaneus) with different modalities (DXA, QUS, and QCT) in a healthy community, especially when there is not any high resolution peripheral quantitative computed tomography (HR-pQCT) and peripheral quantitative computed tomography (pQCT) available.

\section{Methods}

Population

A total of 40 males and females aged 30-60 years were included as healthy volunteers. The healthiness of the volunteers was investigated to ensure they had no history of metabolic bone disease and had not received bone chronic treatment.

\section{Inclusion Criteria}

All participants had a detailed history, so it allowed to exclude secondary symptoms of osteoporosis, such as premenopausal estrogen deficiency and endocrinopathies (eg, hyperthyroidism and Cushing's syndrome), eating disorders, gastrointestinal diseases (eg, celiac), mineral metabolism dysfunction (eg, osteomalacia), and taking medication (eg, glucocorticoids). Women who were pregnant or breast-feeding for the last 12 months were excluded. After confirming the volunteers' health by a specialist, they were instructed and informed on how the test was going to be conducted. All volunteers filled written informed consent forms, which had been approved by the ethics committee of Iran University of Medical Sciences. Participation was voluntary and the participants were informed they could leave the study at any time. To ensure participants' health, blood test was done to measure fasting bloodsugar (FBS), complete blood count $(\mathrm{CBC})$, calcium $(\mathrm{Ca})$, phosphor $(\mathrm{Ph})$, thyroid stimulating hormone (TSH), creatinine, and ferritin.

\section{Areal bone mineral density ( $A B M D$ )}

Dual energy X-ray absorptiometry (DXA) is used to assess the mineral content of bones that are most sensitive to fracture risk. To measure this value, the participants were scanned to find whether they were in the normal category defined by WHO (aBMD; T score $\geq-1.0)(8,9)$. Therefore, the same technician measured aBMD for $\mathrm{L}_{1}$ $\mathrm{L}_{4}$ spine, proximal femur, and forearm by DXA (discovery W, Hologic Inc., Waltham, MA) for all participants at the Endocrinology and Metabolism Research Institute (Tehran University of Medical Sciences).

\section{Quantitative ultrasound (QUS)}

Ultrasound is a mechanical wave used for bone assessment (quantity and quality). The possibility of having a portable, nonionizing and space-saving device encouraged the researchers to use QUS along with other modalities for bone quantification of the weight-bearing bone (calcaneus) (10). Ultrasound measurements of the calcaneus were performed with an Achilles Plus device (GE Medical Systems Lunar, Madison, WI). This modality provided ultrasound parameters as follows: speed of sound (SOS), broadband ultrasound (BUA), and stiffness index (SI), which were derived from BUA and SOS. These parameters were determined by bone's features (eg, BMD, elasticity, and bone architecture) in theory and experience (11).

\section{Volume bone mineral density (VBMD)}

Multidetector computed tomography (MDCT) images of the distal tibia (38\%) (Brilliance, Philips Medical Systems, Amsterdam, Netherlands) were acquired in the hospital for all participants. Hybrid iterative reconstruction algorithm (iDose - level 7) was used which allowed using a low dose of radiation. Other $\mathrm{CT}$ parameters were as follow: spiral acquisition, $90 \mathrm{kVp}, \sim 30$ effective mAs, $3 \mathrm{~mm}$ section thickness, and $150 \mathrm{~mm} * 150 \mathrm{~mm}$ FOV, which was reconstructed across a 1024 matrix size $(0.15 * 0.15 \mathrm{~mm}$ pixel size), 0.8 pitch factor, YC kernel, and $16 \times 0.7 \mathrm{~mm}$ nominal collimation. The left leg of the participants and phantom were scanned simultaneously. The phantom consisted of 3 different concentrations (50, 100 and 200 $\mathrm{mg} \mathrm{K}_{2} \mathrm{HPO}_{4} / \mathrm{cm} 3$ ), which resembled the attenuation behavior of the bone tissue. The computed tomography (CT) values for tibia were converted to BMD scale using the calibration phantom. The imaging protocol was evaluated previously (12).

Since computed tomography acquires cross sectional images, bone density in the trabecular part and cortical part can be evaluated separately. The cortical vBMD, trabecular vBMD, and integral density in $\mathrm{mg} / \mathrm{cm}^{3} \mathrm{~K}_{2} \mathrm{HPO}_{4}$ were calculated as the volume average mineral density within the cortical and trabecular regions and both, respectively. ImageJ (13) software was

\section{Statistical analysis}

Kolmogorov-Smirnov was used to test the normality assumption of the parametric tests. To plot the calibration curve for QCT, the linear regression was used in Excel (Office 2017). Mean and standard deviation (SD) were used for manual segmentation. given as descriptive statistics. The relationships between parameters were analyzed by bivariate (Pearson) correlation (r) analysis. Moreover, Independent samples t test was used to assess differences (significant) in age, weight, height, and BMI parameters between the 2 genders. All statistical tests were 2-sided and performed with alpha $=0.05$ in SPSS (SPSS Inc. Chicago, IL, Version 22.0 for Windows).

\section{Results}

A total of 40 Iranian_healthy volunteers (18 male /22 
females) with normal blood test parameters and aBMDs (in 3 common sites of the body) participated in this study. There were no significant differences between age and BMI between males and females. The participants' demographic characteristics are presented in Table 1.

The mean and standard deviation (SD) measurements are presented in Table 2. In addition, Bivariate (Pearson) correlations (r) between different anatomical sites via multimodal measurements are listed in Table 3. The correlation coefficients between DXA and QUS modalities showed relatively strong correlations between SOS and Ward's aBMD ( $\mathrm{r}=0.608, \mathrm{p}<0.001)$, SI ${ }^{-}$and $\mathrm{L}_{1}-\mathrm{L}_{4}$ aBMD $(\mathrm{r}=0.658, \mathrm{p}<0.001)$ and $\mathrm{BUA}$ and $\mathrm{L}_{1}-\mathrm{L}_{4} \mathrm{aBMD}(\mathrm{r}=0.598$, $\mathrm{p}<0.001)$. There was no correlation among MDCT measures and central bone values and QUS parameters. However, aBMD of $33 \%$ radius and trabecular vBMD in $38 \%$ distal tibia showed weak but significant relationship between peripheral bones $(\mathrm{r}=-0.342, \mathrm{p}=0.044)$.

\section{Discussion}

The limitation to apply all modalities for a specific bone motivated us to use various modalities for different skeletal sites under the assumption that the systemic alteration occurs through the whole skeletal system. Several studies have investigated the correlations between bone measurements among multiple skeletal sites. Cohen et al compared bone strength features between iliac crest bone biopsies (by 2D quantitative histomorphometry and Micro-

Table 1. The participants' demographic features (The mean and standard deviation

\begin{tabular}{|c|c|c|c|c|}
\hline Parameter & Male & Female & Total & $\mathrm{p}$ \\
\hline Age (y) & $42.31(8.26)$ & $45.43(8.50)$ & $44.02(8.43)$ & 0.249 \\
\hline Weight (kg) & $85.06(11.42)$ & $65.85(10.15)$ & $74.48(14.39)$ & 0.001 \\
\hline Height (m) & $1.76(0.08)$ & $1.608(0.7)$ & $1.68(0.11)$ & 0.001 \\
\hline BMI $\left(\mathrm{kg} / \mathrm{m}^{2}\right)$ & 27.(03) & $25.00(3.25)$ & $25.91(3.03)$ & 0.34 \\
\hline
\end{tabular}

Table 2. Mean and Standard Deviation (SD) of multi-modal measurements for different anatomical sites

\begin{tabular}{|c|c|c|c|}
\hline Modality & Site & Measurement (unit) & Mean (SD) \\
\hline \multirow[t]{3}{*}{ QCT } & \multirow[t]{3}{*}{ Tibia } & Cortical vBMD (mg/cm $\left.{ }^{3}\right)$ & $1592.48(38.78)$ \\
\hline & & Trabecular vBMD (mg/cm $\left.{ }^{3}\right)$ & $335.60(79.26)$ \\
\hline & & Integral vBMD $\left(\mathrm{mg} / \mathrm{cm}^{3}\right)$ & $1928.08(95.69)$ \\
\hline \multirow[t]{3}{*}{ QUS } & \multirow[t]{3}{*}{ Calcaneus } & $\operatorname{SOS}(\mathrm{m} / \mathrm{s})$ & $1563.92(39.65)$ \\
\hline & & SI & $103.80(20.38)$ \\
\hline & & BUA (dB/MHz) & $125.43(14.16)$ \\
\hline \multirow[t]{6}{*}{ DXA } & \multirow[t]{2}{*}{ Spine } & L1-L4 aBMD (g/cm $\left.{ }^{2}\right)$ & $1.076(0.14)$ \\
\hline & & Ward's aBMD $\left(\mathrm{g} / \mathrm{cm}^{2}\right)\left(\mathrm{g} / \mathrm{cm}^{2}\right)$ & $0.666(0.14)$ \\
\hline & \multirow{2}{*}{ Femur } & Proximal Total aBMD $\left(\mathrm{g} / \mathrm{cm}^{2}\right)$ & $0.996(0.14)$ \\
\hline & & $\operatorname{Neck}$ aBMD $\left(\mathrm{g} / \mathrm{cm}^{2}\right)\left(\mathrm{g} / \mathrm{cm}^{2}\right)$ & $0.846(0.14)$ \\
\hline & \multirow[t]{2}{*}{ Forearm } & $\mathrm{UD}$ aBMD $\left(\mathrm{g} / \mathrm{cm}^{2}\right)$ & $0.487(0.09)$ \\
\hline & & $33 \%$ Distal Radius aBMD $\left(\mathrm{g} / \mathrm{cm}^{2}\right)$ & $0.710(0.08)$ \\
\hline
\end{tabular}

Table 3. Bivariate (Pearson) correlation (r) for multi-modal measurements

\begin{tabular}{|c|c|c|c|c|c|c|c|c|c|c|c|c|}
\hline Variable & BUA & SOS & SI & $\begin{array}{l}\text { Ward's } \\
\text { aBMD }\end{array}$ & $\begin{array}{c}\text { Proxi- } \\
\text { mal Fe- } \\
\text { mur } \\
\text { aBMD }\end{array}$ & $\begin{array}{l}\text { L1-L4 } \\
\text { aBMD }\end{array}$ & $\begin{array}{l}\text { Neck Fe- } \\
\text { mur } \\
\text { aBMD }\end{array}$ & $\begin{array}{c}\text { UD } \\
\text { Radius } \\
\text { aBMD }\end{array}$ & $\begin{array}{c}33 \% \\
\text { Radius } \\
\text { aBMD }\end{array}$ & $\begin{array}{c}\text { Trabecu- } \\
\text { lar } \\
\text { vBMD }\end{array}$ & $\begin{array}{l}\text { Cortical } \\
\text { vBMD }\end{array}$ & $\begin{array}{l}\text { Integral } \\
\text { vBMD }\end{array}$ \\
\hline
\end{tabular}

\begin{tabular}{lc}
\hline BUA & 1 \\
SOS & 544
\end{tabular}

SOS $\quad .544^{* *}$

SI $\quad .795^{* *}$

Ward's $\quad 443^{* *} \quad .608^{* *} \quad .535^{* *}$

aBMD

Proximal $.516^{* *} \quad .545^{* *} \quad .608^{* *} \quad .792^{* *}$

Femur

$\mathrm{L}_{1}-\mathrm{L}_{4}$

aBMD

Neck Fe-

mur aBMD

UD Radius

$33 \%$ Radius

Trabecular

vBMD

Cortical

vBMD

Integral

$598^{* *}$

$.608 \quad .792$

1

$.521^{* *} \quad .536^{* *} \quad .594^{* *} \quad .828^{* *}$

$.636^{* *} \quad 1$

$\mathrm{vBMD}$

\section{$\begin{array}{llllll}526^{* *} & .445^{* *} & .546^{* *} & .549^{* *} & .665^{* *} & .613^{* *}\end{array}$}

$$
\begin{array}{llll}
.351^{*} & .529^{* *} & .470^{* *} & .335^{*}
\end{array}
$$

$0.262 \quad 0.293$

$0.03 \quad 0.041$

0.223

0.003

1

$0.159 \quad 1$

$\begin{array}{llll}-0.12 & -0.047 & -0.042 & 0.035\end{array}$

$\begin{array}{lll}-0.07 & 0.212 & 0.109\end{array}$

$0.195 \quad-0.084$

$0.114 \quad-0.093$

$-0.056$

$-.342^{*}$

$\begin{array}{lll}0.46 & 0.047 & 0.009\end{array}$

$\begin{array}{lll}0.11 & -0.009 & 0.08\end{array}$

$-0.035$

$-0.038$

0.175

0.223

1

**Correlation is significant at the 0.010 level (2-tailed). * Correlation is significant at the 0.050 level (2-tailed). 
$\mathrm{CT}$ ) with the results of HR-pQCT for the distal of radius and tibia. They reported moderate and significant correlations between the peripheral and central (trans-iliac biopsy) values of microstructure and mechanical features (14). Eckestein et al characterized bone microstructure in in vitro by applying micro-CT for multiple sites, including the neck and trochanter of the femur, Iliac crest, distal radius calcaneus, and lumbar spines (15). Moreover, Liu et al used DXA, QCT, (central), HR-pQCT, and imagebased finite element analyses (FEA) in premenopausal women to evaluate associations among cortical and trabecular bone density, geometry, microstructure, and stiffness of the central and peripheral sites. Significant correlations were found between the stiffness of the distal radius and distal tibia $(r=0.86)$, femur and lumbar spines $(\mathrm{r}=0.49)$, and between peripheral and central bones $(\mathrm{r}=0.56-0.70)(16)$. To the best of our knowledge, most of the studies were performed with $\mu \mathrm{CT}$, HR-pQCT, and pQCT, not MDCT, which is common in medical centers.

The correlation between central and peripheral bones in Table 3 demonstrated that for QUS parameters, among all variables, there was a medium correlation between SOS and $\mathrm{L}_{1}-\mathrm{L}_{4}$ aBMD $(\mathrm{r}=0.558)$, proximal femur aBMD $(\mathrm{r}=0.545)$, and Ward's (the lowest mineral site in proximal of femur) aBMD ( $r=0.608, p<0.001)$. Not only these correlations were not excellent, but also they were significant which is in agreement with other published studies. Based on Clo et al study, the correlation of QUS and DXA in different body sites was not strong enough to replace DXA with QUS, but both parameters allow better access to the bone quantitative and qualitative properties (17-21).

BUA had the highest correlation with L1-L4 aBMD $(\mathrm{r}=0.598, \mathrm{p}<0.001)$ and moderate correlation with proximal femur $(\mathrm{r}=0.516)$ and neck of the femur aBMD $(r=0.521, p=0.001)$. The results were similar to those of Wang et al study in which QUS, DXA, and pQCT were assessed in 258 girls aged 10-13 years in a 2-year longitudinal study (There was a moderate correlation between the calcaneus and femoral neck $(r=0.5, p=0.001)$ and lumbar spines $(\mathrm{r}=0.6, \mathrm{p}=0.001)$. The high content of trabecular bone in both calcaneus and spine may be due to a greater correlation in these areas. However, the diagnosis of osteoporosis in the lumbar spine via QUS of the phalanx was better than the femur region at that study (17). In addition, SI, which is a combination of BUA and SOS, demonstrated more correlation to central aBMDs. Since the role of QUS in the diagnosis and treatment of osteoporosis remains unclear but calcaneus is a weight-bearing bone, it has been recommended as a formative bone to evaluate. In Larijani et al study in which they used the same QUS device, T-score of -1 was proposed as a suitable cutoff level for QUS studies. Also the low agreement between QUS and DXA (Lunar Corporation, Madison, WI) measurements in healthy women was observed (18).

The correlation between the ultradistal of radius and proximal of femur and $\mathrm{L}_{1}-\mathrm{L}_{4}$ aBMDs was reported as $\mathrm{r}=0.665$ and $\mathrm{r}=0.613(\mathrm{p}<0.001)$, respectively. These relatively high correlations were related to the presence of high spongy bone in ultradistal of radius and central bones. In this study, no high correlation was found for
$33 \%$ distal with central aBMDs, while the correlation between 33\% distal radius and hip plus spines was 0.69 in Amiri et al, and it was about 0.60 in Patel et al study. Patel et al have recommended using radius densitometry instead of hip and spine. However, the diagnostic application of forearm densitometry with a lower threshold of about -2.1 instead of -2.5 (recommended by WHO) was suggested $(19,20)$. There was no correlation among MDCT measurements and central bone values. These results might have been due to the cortical declaration in the peripheral site (eg, 38\% distal tibia and 33\% distal radius) rather than a spongy bone loss in central sites.

With regards to the correlation in the peripheral sites, radius 33\% distal aBMD and trabecular vBMD in 38\% distal tibia, the correlation was weak and negative but significant $(\mathrm{r}=-0.342, \mathrm{p}=0.044)$. The negative direction might have been due to the increase of the trabecular vBMD because of trabecularisation of high-density adjacent cortical site. In peripheral sites, approximately $50 \%$ of the cortical bone loss is the consequence of remodeling within the cortical part of the bone close to the marrow. This phenomenon reduces cortical minerals and trabeculitises the cortical, resulting in an overestimation of the density in the trabecular segment (21). The relationship between 33\% distal and ultradistal radius aBMD with QUS parameters was moderate. In a similar study that used pQCT, Hung et al compared pQCT measurements of bone density at distal of tibia and radius to calcaneal (QUS findings) in healthy women and showed weak to moderate correlation $(\mathrm{r}=0.21-0.447)$ between mentioned anatomical sites. Assessment of BUA and velocity of sound (VOS) in calcaneus showed a better correlation to trabecular BMD than cortical BMD and integral BMD in both distal of tibia and radius. More trabecular tissue than cortical (only a shell in the outer layer of the calcaneus bone) can explain the higher correlation of QUS findings to trabecular vBMD (22).

The correlation between 2 central sites (DXA-based measurement), $\mathrm{L}_{1}-\mathrm{L}_{4}$ and proximal femur aBMDs $(r=0.636, p<0.001)$, was good and relatively high for ward's aBMD $(r=0.731, p<0.001)$ and spines measurement, which seems to be due to high trabecular bone in the spines, which is similar to the ward's region with only trabecular bone.

This study also had several limitations. First, the number of participants was small and their age range was limited. Second, lumbar spine and proximal femur vBMDs were not available due to avoiding exposing the volunteers to high radiation dose in central-QCT. Applying state-of-the-art MRI ( $\mu$ MRI) and HRCT (based on iterative reconstruction (IR) algorithm and advanced detectors) techniques for the central skeletal in the near future $(23,24)$ may provide better insight. Thirdly, comparison between bones based on sex with this approach on a larger human sample is highly recommended, especially with a focus on the effect of estrogen deficiency for menopause women.

\section{Conclusion}

The bivariate correlation between the multimodal 
measurements showed systemic bone alteration in the bony skeleton in healthy volunteers. Due to systemic alterations in the bony skeleton, applying peripheral sites quantification with the state-of-the-art MDCT ultralow dose reconstruction is suggested for bone assessment in future studies.

\section{Acknowledgments}

This study was funded by Iran University of Medical Sciences (Grant \# 96-0130-29826).

The authors would like to thank the colleagues at the endocrinology and metabolism research institute (Tehran University of Medical Sciences) for their technical assistance and cooperation.

\section{Conflict of Interests}

The authors declare that they have no competing interests.

\section{References}

1. Budhia S, Mikyas Y, Tang M, Badamgarav E. Osteoporotic fractures: a systematic review of U.S. healthcare costs and resource utilization. Pharmaco Econom. 2012;30(2):147-70.

2. Kanis JA, Johnell O, De Laet C, Jonsson B, Oden A, Ogelsby AK. International variations in hip fracture probabilities: implications for risk assessment. J Bone Miner Res. 2002;17(7):1237-44.

3. Kanis J, Johnell O, Oden A, De Laet C, Jonsson B, Dawson A. Ten-year risk of osteoporotic fracture and the effect of risk factors on screening strategies. Bone. 2002;30(1):251-8.

4. Ettinger MP. Aging bone and osteoporosis: strategies for preventing fractures in the elderly. Arch Intern Med. 2003;163(18):2237-46.

5. Lespessailles E, Chappard C, Bonnet N, Benhamou CL. Imaging techniques for evaluating bone microarchitecture. Joint bone spine. 2006;73(3):254-61.

6. Zebaze RM, Ghasem-Zadeh A, Bohte A, Iuliano-Burns S, Mirams M, Price RI, et al. Intracortical remodelling and porosity in the distal radius and post-mortem femurs of women: a cross-sectional study.Lancet 2010;375(9727):1729-36.

7. Abdelmohsen AM. Comparison of central and peripheral bone mineral density measurements in postmenopausal women. Journal of chiropractic medicine. 2017;16(3):199-203.

8. Organization WH. Assessment of fracture risk and its application to screening for postmenopausal osteoporosis: report of a WHO study group [meeting held in Rome from 22 to 25 June 1992]. 1994.

9. Siris ES, Chen Y-T, Abbott TA, Barrett-Connor E, Miller PD, Wehren $\mathrm{LE}$, et al. Bone mineral density thresholds for pharmacological intervention to prevent fractures.Arch Intern Med. 2004;164(10):110812.

10. Pisani P, Renna MD, Conversano F, Casciaro E, Muratore M, Quarta E, et al. Screening and early diagnosis of osteoporosis through X-ray and ultrasound based techniques. World J Radiol. 2013;5(11):398.

11. Hans D, Wu C, Njeh C, Zhao S, Augat P, Newitt D, et al. Ultrasound velocity of trabecular cubes reflects mainly bone density and elasticity. Calcif Tissue Int. 1999;64(1):18-23.

12. Malekzadeh M, Abbasi-Rad S, Keyak J, Nabil M, Asadi M, Mobini $\mathrm{N}$, et al. Liquid Calibration Phantoms in Ultralow-Dose QCT for the Assessment of Bone Mineral Density. J Clin Densitom. 2019.

13. Schneider CA, Rasband WS, Eliceiri KW. NIH Image to ImageJ: 25 years of image analysis. Br J Pharmacol. 2012;9(7):671.

14. Cohen A, Dempster D, Müller R, Guo X, Nickolas T, Liu X, et al. Assessment of trabecular and cortical architecture and mechanical competence of bone by high-resolution peripheral computed tomography: comparison with transiliac bone biopsy. Osteoporos Int. 2010;21(2):263-73.

15. Eckstein F, Matsuura M, Kuhn V, Priemel M, Müller R, Link TM, et al. Sex differences of human trabecular bone microstructure in aging are site-dependent. J Bone Miner Res. 2007;22(6):817-24.

16. Liu XS, Cohen A, Shane E, Yin PT, Stein EM, Rogers H, et al. Bone density, geometry, microstructure, and stiffness: Relationships between peripheral and central skeletal sites assessed by DXA, HR-pQCT, and cQCT in premenopausal women. J Bone Miner Res. 2010;25(10):222938.

17. Hamidi Z, Sedaghat M, Hejri SM, Larijani B. Defining cut-off values for the diagnosis of osteoporosis in postmenopausal women by quantitative ultrasonography of the phalanx. Gynecol Endocrinol. 2008;24(10):546-8

18. Larijani B, Dabbaghmanesh MH, Aghakhani S, Sedaghat M, Hamidi Z, Rahimi E. Correlation of Quantitative Heel Ultrasonography With Central Dual-Energy X-ray Absorptiometric Bone Mineral Density in Postmenopausal Women. J Ultrasound Med. 2005;24(7):941-6.

19. Patel R, Blake GM, Fogelman I. An evaluation of the United Kingdom National Osteoporosis Society position statement on the use of peripheral dual-energy X-ray absorptiometry. Osteoporos Int. 2004;15(6):497-504.

20. Amiri L, Kheiltash A, Movassaghi S, Moghaddassi M, Seddigh L. Comparison of Bone Density of Distal Radius With Hip and Spine Using DXA. Acta Medica Iranica. 2017:92-6.

21. Odetti P, Rossi S, Monacelli F, Poggi A, Cirnigliaro M, Federici M, et al. Advanced glycation end products and bone loss during aging. Ann New York Acad Sci. 2005;1043(1):710-7.

22. Hung VWY, Qin L, Au SK, Choy WY, Leung KS, Leung PC, et al. Correlations of calcaneal QUS with pQCT measurements at distal tibia and non-weight-bearing distal radius. $\mathrm{J}$ Bone Miner Metabol. 2004;22(5):486-90.

23. Gupta R, Cheung AC, Bartling SH, Lisauskas J, Grasruck M, Leidecker C, et al. Flat-panel volume CT: fundamental principles, technology, and applications. Radio Graphics. 2008;28(7):2009-22.

24. Krug R, Banerjee S, Han E, Newitt D, Link T, Majumdar S. Feasibility of in vivo structural analysis of high-resolution magnetic resonance images of the proximal femur. Osteoporos Int. 2005;16(11):1307-14 